\title{
Bioindication-based estimates as an integral part of the environment quality assessment
}

\author{
A. Oudalova ${ }^{1}$, S. Geras'kin ${ }^{1}$, V. Dikarev¹, N. Dikareva1', G. Kozmin², \\ B. Michalik ${ }^{3}$ and M. Wysocka ${ }^{3}$ \\ ${ }^{1}$ Russian Institute of Agricultural Radiology and Agroecology, 249032 Obninsk, Russia \\ ${ }^{2}$ State Technical University of Atomic Energy, 249040 Obninsk, Russia \\ ${ }^{3}$ Central Mining Institute, PI. Gwarkow 1, 40-166 Katowice, Poland
}

\begin{abstract}
A complex approach for the environment health assessment integrating information on contaminants levels registered in environment compartments and bioindication-based estimates is developed and applied to two studies carried out at sites with different scenarios of long-term impact. The environment state is assessed at the territory of a nuclear waste storage facility, Obninsk, Russia, and in the post-mining areas of the Upper Silesia, Poland with enhanced levels of natural radioactivity. Concentrations of micro-, macroelements and appropriate radionuclides activities are measured in samples of water and sediment. With the Allium cepa plant system, phyto- and genotoxicity of the sampled water and soil is estimated to assess a hazardous potential of the complex contamination. As biological endpoints, frequency and spectrum of chromosome aberrations and mitotic abnormalities in ana-telophase cells as well as mitotic activity in Allium cepa root tips are used. An approach and techniques are developed to reveal factors and contamination components that play a governing role in an induction of the observed biological effect and estimate their relative contribution. Compatibility of findings observed and legislative standards adopted are discussed. From the studies carried out, the Allium-test proved to be a sensitive and simple bioassay efficient in genotoxic monitoring of environmental pollutants.
\end{abstract}

\section{INTRODUCTION}

Anthropogenic nuclear and industrial activities can substantially increase environmental exposure levels. A great deal of research have been carried out to specify and justify constraints for chemical substances release and radionuclide discharges into the environment, and regulatory decision-making strategies are still an important topic for the human health and ecosystem sustainability protection. Contamination of territories through human civil and military activities such as nuclear facilities operation, mining sites, nuclear weapon testing involves, however, a complex impact so that there are a number of situations where a mixture of radionuclides is supplemented by other potentially hazardous substances (e.g. heavy or alkali metals). There is still a large lack of knowledge on actual hazard of such combined contamination and a need to fill the gap between an acquisition of spectrum and levels of environmental pollutants to possible adverse effects to biota and human health.

This study was aimed at developing a complex approach for the environment health assessment integrating information on contaminants levels registered in the environment compartments (water and sediment) and bioindication-based estimates of adverse effects from their combination.

\section{MATERIALS AND METHODS}

Sampling is made at two study sites illustrating different scenarios of long-term impact. The first one is the territory of a nuclear waste storage facility (NWSF), Obninsk, Russia. Water samples were collected in 2004 from monitor wells as well as from nearby water bodies. Detailed description of the study, radionuclide activities and chemicals concentrations in the samples is given in [1]. The second is 
post-mining areas of the Upper Silesia, Poland [2] with enhanced levels of natural radioactivity where water and sediment were sampled in 2005 from underground galleries of collieries and the Gostynka River and ponds impacted. Concentrations of micro-, macroelements and appropriate radionuclides activities are measured for all sampling points with routine techniques.

To estimate a hazardous potential of the complex contamination in the study sites for biological objects, the Allium-test is applied to assess cyto- and genotoxicity of sampled water and soil for plant system. As biological endpoints, frequency of aberrant cells (AC) in ana-telophase cells and mitotic index (MI) in Allium cepa root tips are used.

To reveal a relationship of biological effect on pollution levels at combined environment contamination, a dimensionality of predictors' space is to be reduced through a selection of the most informative variables that can describe a dependence of the resultant variable (biological effect) in the best way. Thus, non-informative predictors that differ little between sampling points are excluded from quantitative analysis. From every group of highly-correlated variables $(r \geq 95 \%, p<0.05)$ only one is taken for further calculations. Then, an approach of the step-by-step inclusion/exclusion (SSIE) of predictors essential for multivariable regression [3] is realized. As a resultant (dependent) variable $Y$, biological effect observed at the Allium-test application, i.e. AC frequency or MI values, is considered. $Y$ contains $N$ values relating to the total number of preparations scored in all corresponding variants. Input matrix of independent variables $M$ is assembled of $\left(N \times p^{\prime}\right)$ units, where $p^{\prime}$ is the number of previously sifted predictors. A predictor is recognized as non-essential, if at its inclusion or exclusion in output matrix $M^{\prime}$, there is no significant loss in data approximation quality quantified through the determination coefficient for multivariate regression, $R^{2}$. The Hayek criterion, $H$ is applied to test the significance of the $R^{2}$ decreasing/increasing.

From the SSIE algorithms application to linear and non-linear regression models, several functions are revealed for every study site, environment compartment and biological effect considered. To choose a model with the best goodness of data fitting, the quality for data approximation with these functions is compared with several most common statistics including the determination coefficients, $R^{2}$, Fisher statistics, $F$, criteria of structural identification, $T$ and the Hayek criterion, $H$.

\section{RESULTS AND DISCUSSION}

\subsection{Nuclear waste storage facility}

Since 1998, there has been detected a presence of ${ }^{90} \mathrm{Sr}$ in water from monitor wells at the NWSF territory due to a leakage in a storage tank. In 2004 , specific activity of ${ }^{90} \mathrm{Sr}$ in most contaminated samples were about 10 times of the intervention level $\mathrm{IL}=5.0 \mathrm{Bk} / \mathrm{kg}$ adopted in Russia for drinking water. Concentrations of some heavy metals ( $\mathrm{Mn}, \mathrm{Zn}, \mathrm{Ni}$ ) exceeded the Russian guideline values for maximum permissible content in waterbodies.

At the onion bulbs germination in the water sampled from the NWSF and nearby territory, the mitotic activity in root meristem changes little (Fig. 1A). The inhibitory effect is observed for water collected from a bog (Variant 3) and distilled water (Variant 2); both these samples are obviously unfavorable for higher plant ontogenesis. Significant stimulation is found in Variant 6 (the NWSF territory).

For cytogenetic disturbances, a transparent effect of water contamination is shown since the AC occurrence in root meristem of Allim cepa is significantly over the control for all water samples taken from and nearby the territory of the NWSF (Fig. 1B), which indicates a potential adverse impact on the natural environment in a vicinity of the NWSF. The main contribution to the genotoxic impact is provided by laggard chromosome occurrence, which frequency significantly enhances the control level in five variants disturbances. Remarkable role is also played by such severe disturbences as chromosome bridges and tripolar mitoses.

Initial data on sampled water quality are presented by $p=21$ indices (radionuclides, chemicals, and some general indices of water quality). After sifting out non-informative and high-correlated variables, 

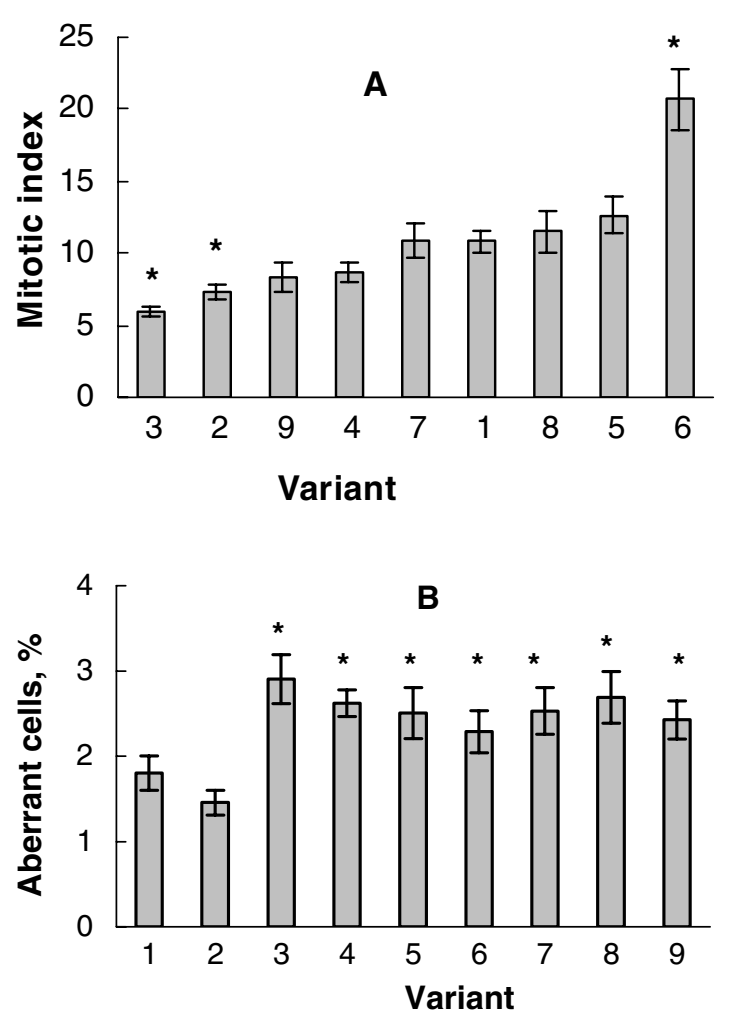

Figure 1. Mitotic index $\left(10^{-2}\right)$, A, and aberrant cell frequency (\%), B, in root tip cells of Allium cepa germinated in waters sampled from the NWSF and nearby territory, (mean \pm se). Variant 1 - natural control, Variant 2 - distilled water; *-difference from Variant $1, \mathrm{p}<0.05$.

the number of essential predictors is reduced down to $p^{\prime}=11$, and for multiple regression analyses ${ }^{90} \mathrm{Sr}$ activity and $\mathrm{K}, \mathrm{Na}, \mathrm{Ca}, \mathrm{Mg}, \mathrm{Fe}, \mathrm{Mn}, \mathrm{Ni}, \mathrm{Cu}, \mathrm{Cr}$, and $\mathrm{Pb}$ concentrations in water are supplied. With the SSIE algorithm application, four linear models with various number of terms (from 1 to 5) are revealed for fitting the data on AC frequency and MI. It is shown [1] that the best of them for approximating the genotoxic effect observed is

$$
Y(\%)=(1.9 \pm 0.2)+(0.2 \pm 0.05) \cdot[\mathrm{Pb}], R^{2}=7.76 \%, p<0.001,
$$

and for cytotoxic effect:

$$
Y\left(10^{-2}\right)=(10.6 \pm 0.8)+(4.3 \pm 0.5) \cdot 10^{-3} \cdot[\mathrm{Mn}]-(1.5 \pm 0.7) \cdot[\mathrm{K}], R^{2}=28.8 \%, p<0.0001,
$$

where chemicals' concentrations are in $\mathrm{mg} / \mathrm{l}$.

\subsection{The Upper Silesian Coal Basin, Poland}

Water samples are taken from five locations, and 27 indices of water quality and pollution level are measured. There found violations of the Polish national and international (WHO) standards for all the samples except for 'natural control'. Onion bulbs appeared not able to grow on these waters because of their extremely high salinity and pollution, and a 20 times dilution of samples from underground galleries and 3 times dilution for water from the Gostynka River were applied to carry out the Allium-test. Even after the dilution, chlorides content and $\mathrm{Na}$ concentrations are over the corresponding 
limits in all non-reference samples, $\mathrm{Mg}, \mathrm{Ba}$, and $\mathrm{Sr}$ concentration limits are violated in Variants $\mathrm{W} 1$ and $\mathrm{W} 2$, and ${ }^{226} \mathrm{Ra}$ and ${ }^{228} \mathrm{Ra}$ activities are over the activity concentration limits in Variant W1.

For sediment, two of four samples collected should be marked as radioactive waste according to the Polish national guidelines, since their ${ }^{224} \mathrm{Ra},{ }^{226} \mathrm{Ra}$ and ${ }^{228} \mathrm{Ra}$ activities are over the corresponding limits. Also, among 12 chemicals measured, Ba concentrations exceed the national concentration limit for soils of industrial, communication and mining areas in all the sediment samples.

Mitotic activity at both water and sediment testing appeared little affected, and is not considered here further. Genotoxic effect found is shown in Fig. 2. Allium cepa bulbs germination on the contaminated water (diluted) and sediment resulted in significance increase in cytogenetic disturbances occurrence in root meristem as compared to the natural control (Variant W4), distilled water (Variant DW), and the reference soil (Variant $\mathrm{C}$ ). In all non-reference sediment and water samples from underground galleries governing contribution to cytogenetic effect is made by chromosome (double) bridges and lagging chromosomes; moreover, structure of the aberration spectrum in water collected from the underground galleries distinguishes from that found for the river, which proves a difference in composition and concentration levels of pollutants in waters originating from various sources.

Similar to the first study, the number of essential predictors is reduced to $p^{\prime}=9$ for water testing $\left(\mathrm{Ba}, \mathrm{Cd}, \mathrm{Hg}, \mathrm{K}, \mathrm{Mn}, \mathrm{Na}, \mathrm{Ni}, \mathrm{Zn}\right.$, and sum of radium nuclides $\mathrm{Ra}_{\Sigma}$ ) and $p^{\prime}=6$ for sediment testing $\left(\mathrm{Ba}, \mathrm{Cu}, \mathrm{Cr}, \mathrm{Zn}, \mathrm{Pb}, \mathrm{Ra}_{\Sigma}\right)$. The SSIE algorithm application to linear and non-linear (taking account for paired interaction of predictors) models resulted in several appropriate functions, as earlier. The best models for describing the data on water and sediment genotoxicity testing are $Y=(1.5 \pm$ $0.1)+(7.4 \pm 0.8) \cdot 10^{-2} \cdot[\mathrm{K}]+(2.7 \pm 0.4) \cdot 10^{-2} \cdot[\mathrm{Ba}]-(38.0 \pm 7.0) \cdot[\mathrm{Zn}], R^{2}=51.5 \%$ and $Y=$ $(11.4 \pm 0.7)+(2.4 \pm 0.2) \cdot 10^{-2} \cdot[\mathrm{Ba}]-(0.4 \pm 0.03) \cdot[\mathrm{Cr}]+(6.7 \pm 0.6) \cdot 10^{-4} \cdot\left[\mathrm{Ra}_{\Sigma}\right], R^{2}=62.2 \%$. In Fig. 3, genotoxicity observed at the water samples testing and estimated from the best model revealed is presented; uncertainties in the extrapolated levels are computed from standard errors of the regression coefficients. It is seen from Fig. 3 that the observed and predicted values are in good accordance.
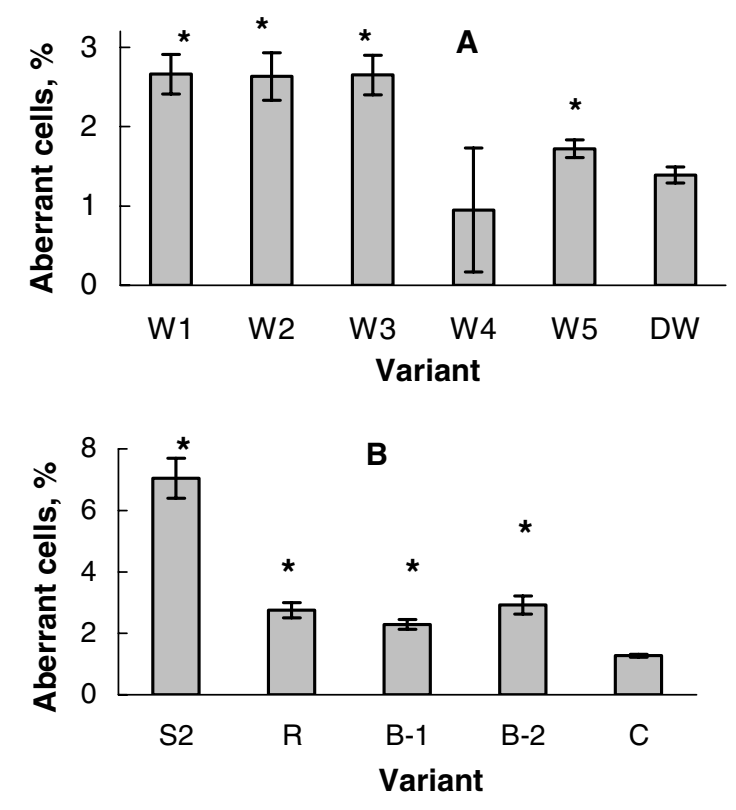

Figure 2. Aberrant cell frequency in root tip cells of Allium cepa germinated at tested water (A) and sediment (B) samples from the Upper Silesia, Poland (mean \pm se); * - difference from Variant W4 (natural control) or Variant C (reference soil), $\mathrm{p}<0.05$. 


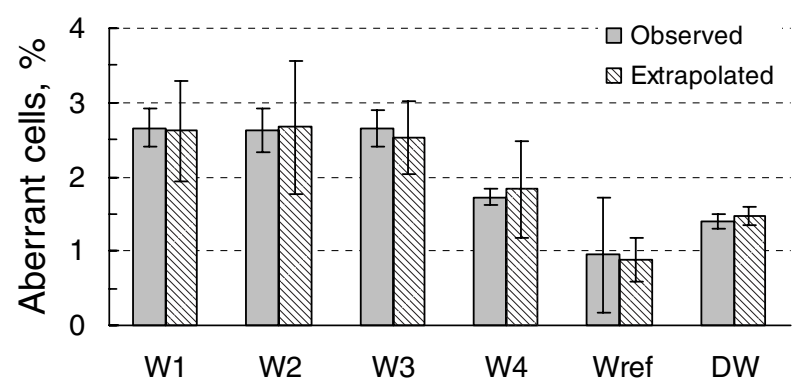

Figure 3. Genotoxicity of the water samples observed and extrapolated with the best regression model.

\section{CONCLUSIONS}

Measurements of dose and concentrations of contaminants could provide a detailed description of pollution levels, but only indirectly indicate potential biological consequences of mixed pollution of the environment. Biological assays, in turn, provide a mechanism by which the biological impact of mixtures can be quantified. A combination of these two techniques allows identifying the major sources of risk which require continuous biological monitoring. Therefore, an adequate assessment of the risk to the environment from contamination needs to be based on the simultaneous use of pollutant chemical control methods and biological tests. As a genotoxicity test, the Allium-based assay of chromosome aberration in anaphase-telophase is for many reasons [4-6] especially useful for the rapid screening of both chemicals and radionuclides that may be involved in environmental hazards. Its high sensitivity ensures that contaminants will not be overlooked, which may be of special importance when complex mixtures are to be tested. Indeed, in both studies carried out in different radioecological conditions, the high levels of genotoxicity are observed in all samples tested, with the important contribution from severe types of cell damage.

The present work and our previous studies [7-9] show that an adequate environment quality assessment cannot rely only on information about pollutants concentrations. Authorities in charge of controlling the levels of industrial load have dealt with limited lists of toxicants. Furthermore, a mutual intensification of the effects from environmental contaminants is very possible, especially when they occur in situations of low-level exposures, as demonstrated earlier [8,9]. It is therefore impossible to estimate the biological risk from a combined exposure if based only on the knowledge of contaminants levels in the environment. This emphasizes the need to update some current principles of ecological standardization, which are still in use nowadays.

Finally, local biologically relevant contaminations of surface and underground water as well as sediments from the Upper Silesia post-mining areas and territory affected by a nuclear waste storage facility, Obninsk, Russia are displayed by means of simultaneous use of chemical control methods and Allium-test. To study relationships between biological effects registered and the environment combined contamination, approaches based on multidimensional mathematical analysis are developed and applied. Key pollutants governing biological effect observed with bioindication approach are revealed. The positive results obtained in the Allium-test should be considered as a warning and also an indication that the tested environments may be of risk to both human health and biological components of natural ecosystems. Complex issues are involved in evaluating environmental risk, and an effectively linking of bioindication screening assays to the well-established environmental pollution monitoring approach is a way of improving and upgrading an existing system of the public and the environment protection to meet requirements of consistency between nowadays scientific knowledge and decision-making process. 


\section{Acknowledgments}

The work was supported by RFBR project no. 05-04-96721, R\&D project no. 371000035-173 and Tok-DEV WaterNorm (contract no. MTKD-CT-2004-003163).

\section{References}

[1] Oudalova A.A., Geras'kin S.A., Dikarev V.G., Dikareva N.S., Krouglov S.V., Vaizer V.I., Kozmin G.V. and Chernonog E.V., Development of methods for a complex assessing ecological state at territories affected by nuclear industry enterprises. Proceedings of the regional competition of the natural-science projects, Issue 9. Kalugian scientific centre, Kaluga, 2006. pp. 221-239 (in Russian).

[2] Geras'kin S.A., Michalik B., Oudalova A.A., Dikareva N.S., Chalupnik S., Dikarev V.G. and Wysocka M., Sci. Tot. Environ. (2008), in preparation.

[3] Aivazyan S.A., Yenyukov I.S. and Meshalkin L.D. Applied Statistics. Study of Relationships. Finansy i statistika, Moscow, 1985.

[4] Grant W.F., Mutat. Res. 99 (1982) 273-91.

[5] Fiskesjo G., Mutat. Res. 197 (1988) 243-60.

[6] Rank J. and Nielsen M.H., Mutat. Res. 418 (1998) 113-19.

[7] Evseeva T.I., Geras'kin S.A. and Shuktomova I.I., J. Environ. Radioact. 68 (2003) 235-48.

[8] Evseeva T.I., Geras'kin S.A., Shuktomova I.I. and Taskaev A.I., J. Environ. Radioact. 80 (2005) 59-74.

[9] Geras'kin S.A., Kim J.K., Dikarev V.G., Oudalova A.A., Dikareva N.S. and Spirin Y.V., Mutat. Res. 586 (2005) 147-59. 